\title{
Non-overlapping and Inverse Associations Between the Sexes in Structural Brain-Trait Associations
}

\author{
Daphne Stam ${ }^{1}$, Yun-An Huang ${ }^{1}$ and Jan Van den Stock ${ }^{1,2,3 *}$ \\ ' Laboratory for Translational Neuropsychiatry, Department of Neurosciences, KU Leuven, Leuven, Belgium, ${ }^{2}$ Geriatric \\ Psychiatry, University Psychiatric Center KU Leuven, Leuven, Belgium, ${ }^{3}$ Brain and Emotion Laboratory, Maastricht \\ University, Maastricht, Netherlands
}

\section{OPEN ACCESS}

Edited by:

Marina A. Pavlova Tübingen University Hospital,

Germany

Reviewed by:

Nicole Anderson,

Brigham Young University,

United States

Robert Kelvin Perkins,

Norfolk State University, United States

${ }^{*}$ Correspondence:

Jan Van den Stock

jan.vandenstock@med.kuleuven.be

Specialty section:

This article was submitted to Gender, Sex and Sexuality Studies,

a section of the journal

Frontiers in Psychology

Received: 30 November 2018

Accepted: 04 April 2019

Published: 24 April 2019

Citation:

Stam D, Huang $Y-A$ and

Van den Stock J (2019)

Non-overlapping and Inverse Associations Between the Sexes in Structural Brain-Trait Associations.

Front. Psychol. 10:904.

doi: 10.3389/fpsyg.2019.00904
Personality reflects the set of psychological traits and mechanisms characteristic for an individual. The brain-trait association between personality and gray matter volume (GMv) has been well studied. However, a recent study has shown that brain structurepersonality relationships are highly dependent on sex. In addition, the present study investigates the role of sex on the association between temperaments and regional GMv. Sixty-six participants (33 male) completed the Temperament and Character Inventory (TCl) and underwent structural magnetic resonance brain imaging. MannWhitney $U$ tests showed a significant higher score on Novelty Seeking (NS) and Reward Dependence $(R D)$ for females, but no significant group effects were found for Harm Avoidance $(\mathrm{HA})$ and Persistence $(\mathrm{P})$ score. Full factor model analyses were performed to investigate sex-temperament interaction effects on GMv. This revealed increased GMv for females in the superior temporal gyrus when linked to NS, middle temporal gyrus for $\mathrm{HA}$, and the insula for RD. Males displayed increased GMv compared to females relating to $P$ in the posterior cingulate gyrus, the medial superior frontal gyrus, and the middle cingulate gyrus, compared to females. Multiple regression analysis showed clear differences between the brain regions that correlate with female subjects and the brain correlates that correlate with male subjects. No overlap was observed between sexspecific brain-trait associations. These results increase the knowledge of the role of sex on the structural neurobiology of personality and indicate that sex differences reflect structural differences observed in the normal brain. Furthermore, sex hormones seem an important underlying factor for the found sex differences in brain-trait associations. The present study indicates an important role for sex in these brain structure-personality relationships, and implies that sex should not just be added as a covariate of no interest.

Keywords: sex, temperaments, voxel-based morphometry, brain-trait association, full factor model

\section{INTRODUCTION}

Some people are almost constantly looking for new challenges, while others choose to stick to old habits. There is a large diversity in the way people behave and how they think. This diversity can be explained by personality, a set of psychological traits and mechanisms characteristic for an individual (Larsen and Buss, 2010). It is well known that personality traits are subject to sex 
differences. For instance, females typically show higher agreeableness and neuroticism compared to males (Chapman et al., 2007; Weisberg et al., 2011). Little is known about the neurobiology that is associated with sex differences in personality traits. However, there are striking differences between the sexes in the neural basis of emotional processes (Kret and De Gelder, 2012), in the relationship between narcissistic personality and regional grey matter volume (GMv; Yang et al., 2015) and findings implicate structural differences as a partial explanation for sex differences in antisocial personality (Raine et al., 2011). A recent study of Nostro et al. (2016) showed that brain structure-personality associations are highly dependent on sex. They used the NEO Five Factor Inventory (NEO FFI) to measure personality and found no significant associations between the NEO FFI (Costa and McCrae, 1992b) and regional (GMv) for the combined (males and females) sample. However, they did find sex-specific associations. Interestingly, significant associations with GMv were detected only in males. For neuroticism negative correlations were found for GMv of parieto-occipital sulcus/cuneus, left fusiform gyrus/cerebellum, and right fusiform gyrus. Positive correlations were found between conscientiousness and GMv of left precuneus and parietooccipital sulcus. Also a positive correlation was found between extraversion and GMv precuneus/parieto-occipital sulcus, thalamus, left fusiform gyrus/cerebellum, and right cerebellum.

The present study addresses sex differences in the neurobiology of temperaments which are based on the psychobiological personality account of Cloninger et al. (1993). Temperaments are regarded to be heritable and homogeneous, stable over time, emerge early in life, and independent of each other (Cloninger, 1986; Cloninger et al., 1993; Heath et al., 1994; Stallings et al., 1996; Comings et al., 2000; Larsen and Buss, 2010). The Temperament and Character Inventory (TCI) assess these temperaments (Cloninger et al., 1993). The TCI contains four temperament scales: (1) novelty seeking (NS); (2) harm avoidance (HA); (3) reward dependence (RD); and (4) persistence (P) (Cloninger et al., 1993). These temperament scales can be further subdivided into different subscales: NS can be divided into exploratory excitability (NS1), impulsiveness (NS2), extravagance (NS3), and disorderliness (NS4); HA is composed of anticipatory worry (HA1), fear of uncertainty (HA2), shyness (HA3), and fatigability (HA4); RD can be divided into sentimentality (RD1), social attachment (RD2), and dependency (RD3); the temperament $\mathrm{P}$ is not further divided (Cloninger et al., 1993).

Several studies have investigated the associations between temperaments and regional GMv (Iidaka et al., 2006; Gardini et al., 2009; Picerni et al., 2013; Laricchiuta et al., 2014; Stam et al., 2018). However, sex is a variable that is typically statistically controlled for and little is known about its effect on temperamentbrain associations.

In the current study, we try to answer the question "how does sex affect the association between temperaments and regional GMv?" In order to answer this question, we investigate the interaction between sex and temperaments (NS, HA, RD, and P) on regional GMv. Not much is known about the relation between sex and the association between temperaments and regional GMv. The study of Nostro et al. (2016) showed a positive correlation between extraversion and GMv precuneus/parietooccipital sulcus, thalamus, left fusiform gyrus/cerebellum, and right cerebellum, in males. As extraversion is a trait known to be linked with NS (Gocłowska et al., 2018), we expect to find comparable results. The aim of the current study is to reveal distinct and common effects between the sexes in associations between regional GMv and temperaments. Furthermore, sex-specific associations between personality and risk for neuropsychiatric disorders have been reported, including eating disorders (Gual et al., 2002; Krug et al., 2009) and mood disorders (Costa and McCrae, 1992a; Afifi, 2007). A better understanding of the neurobiology of sex differences in personality temperaments may hold benefits for development of sex-specific treatments of those disorders.

\section{MATERIALS AND METHODS}

This study was approved by the Ethical Committee of University Hospitals Leuven. All subjects gave written informed consent in accordance with the Declaration of Helsinki.

\section{Participants}

Sixty-six healthy subjects participated, 33 males (mean age $\pm \mathrm{SD}=38 \pm 13$ years, range 21-75) and 33 females (mean age $\pm \mathrm{SD}=36 \pm 11$ years, range 21-65) ${ }^{1}$. Mann-Whitney $U$ tests showed that no significant sex differences were detected for age $(P=0.568)$. The sample was a non-clinical population composed of three subgroups to increase the variability of the loadings on personality scales: (1) Fourteen participants with premanifest Huntington's disease [21\% (50\% male)], (2) Eighteen gene-negative controls from Huntington's disease families [27\% (50\% male)], (3) thirty-four healthy controls [52\% (50\% male)]. We included premanifest Huntington's disease subjects, referring to the absence of motor symptoms in combination with a positive mutation status. In addition, a radiologist evaluated the structural scans and there were no abnormalities at the individual level. As the main question of the current article focusses on sex differences, observing possible group differences of the three subgroups falls outside the scope of this article. The different subgroups are merely added for methodological reason (increasing the variability in the dataset).

\section{Temperament and Character Inventory}

Temperament and character inventory is a questionnaire for measuring seven domains of personality and consists of 240dichotomous items. The seven domains are divided in three character scales (self-directedness, cooperativeness, and selftranscendence) and four temperament scales (NS, HA, RD, and $\mathrm{P})$. A validated Dutch translation was used with reasonable to good psychometric internal consistency (Cronbach's $\alpha$ range, 0.64-0.87). It was validated in a representative sample of Dutch individuals $(n=1034)$ (version 1.3; Datec Psychological Tests,

${ }^{1}$ All data is available through the corresponding author (jan.vandenstock@med.kuleuven.be). 
Leiderdorp, Netherlands). NS reflects enthusiasm, impulsivity, and reward-sensitivity (e.g., "I like to explore new ways to do things"); HA is related to acting with caution and passive avoidance behavior (e.g., "I often feel tense and worried in unfamiliar situations, even when others feel there is little to worry about"); RD is associated with responsiveness to signals of reward (e.g., "I like to please other people as much as I can") and $\mathrm{P}$ indicates motivation without direct external reward (e.g., "I am more of a perfectionist than most people") (Cloninger, 1986; Cloninger et al., 1993; Larsen and Buss, 2010; Laricchiuta et al., 2014).

\section{MRI Acquisition}

Neuroimaging was performed on a 3T MRI scanner. A highresolution T1-weighted anatomical image (voxel size: $0.98 \mathrm{~mm} \times 0.98 \mathrm{~mm} \times 1.20 \mathrm{~mm}$ ) was acquired on a single 3 T Philips Achieva system equipped with a 32 channel head coil using a 3D turbo field echo sequence (TR:9.6 ms; TE:4.6 ms; matrix size: $256 \times 256$; 182 slices).

\section{Structural Data Analysis}

Data was analyzed using CAT12, a Computational Anatomy Toolbox $^{2}$ (Gaser and Kurth, 2017) running under SPM12 ${ }^{3}$ and MATLAB (R2016b). In order to investigate the role of sex in the association between regional GMv and temperaments, we performed voxel-based morphometry (VBM). Preprocessing consisted of normalization to MNI space, tissue classification (segmentation) into GM, white matter (WM), and cerebrospinal fluid (CSF), and bias correction of intensity non-uniformities. The amount of volume changes due to spatial registration were scaled, in order to retain the original local volumes (modulating the segmentations). The modulated images were smoothed using a $12 \mathrm{~mm} \times 12 \mathrm{~mm} \times 12 \mathrm{~mm}$ full-width at half-maximum Gaussian kernel.

\section{Statistical Analysis}

In order to investigate sex differences in both the main scales (NS, HA, RD, and P) as in the subscales (NS1, NS2, NS3, NS4, HA1, HA2, HA3, HA4, RD1, RD2, RD3, and P) statistical tests were preceded by a normality check on the distributions of the respective residuals by means of Shapiro-Wilk test. In case normality could not be assumed, non-parametric tests were performed. For the purpose of uniformity of analyses, we performed parametric tests or non-parametric tests on all behavioral data.

To investigate sex differences in brain-temperament associations, we first performed a full factorial model analysis on the voxelwise GMv of the total sample, to observe the interaction effect between sex and the temperament scores (NS, HA, RD, and $\mathrm{P}$ ). Sex was included as factor and the temperament scores as interaction with the factor. Age and total intracranial volume (TIV) were included as variables of no interest.

Eight contrasts were performed: for every temperament scale, we investigated the male and female association. In every analysis

\footnotetext{
${ }^{2}$ http://www.neuro.uni-jena.de/cat/

${ }^{3}$ http://www.fil.ion.ucl.ac.uk/spm/software/spm12/
}

we added the remaining temperament scores as variables of no interest, in order to maximize the specificity of the results of a single temperament (as it controls for the association that is contained by the other temperaments).

Secondly, multiple regression analyses were performed on the smoothed GM-images for males and females separately. The four temperament scores (NS, HA, RD, and P) were entered as regressors in a single model, in addition to age, and TIV, which were included as variables of no interest. In total eight contrasts were performed on the male data and eight contrasts on the female data.

To investigate any overlap between the sex-selective results, we inclusively masked the results of the regression analysis of both groups. The statistical threshold was set at a $P_{\text {height }}<0.001$ $(k=10)$ in combination with $P_{\text {height }}<0.05$ FWE-corrected following Small Volume Correction.

Anatomic labeling of significant clusters was performed using $\mathrm{xjView}^{4}$ and clusters were visualized using MRICron ${ }^{5}$.

\section{RESULTS}

Shapiro-Wilk test showed that residuals of NS, HA, RD, and $\mathrm{P}$ were normally distributed $(P>0.108)$. The residuals of the different subscales, however, were not normally distributed. For the purpose of uniformity of analyses, we performed nonparametric tests on all behavioral data. Mann-Whitney $U$ tests showed a significantly higher score on NS $(p=0.02)$ and RD $(p<0.001)$ for females, but no significant group effects were found for the HA and $\mathrm{P}$ score $(P>0.445)$. Furthermore, Mann-Whitney $U$ tests showed sex differences between the subscales (Figure 1).

\section{Interaction Between Sex and Temperaments in Voxel-Wise GMv}

To investigate the role of sex in the association between regional GMv and temperaments, we ran a full factorial model on the smoothed GM images. This revealed interactions between sex and temperaments. The results are presented in Table 1. Males and females show opposite associations between NS and GMv in the superior temporal gyrus (females show positive association; $t=4.96, p=0.001$ ), for HA in the middle temporal gyrus (females show positive association; $t=4.56, p=0.003$ ), for $\mathrm{RD}$ in the insula (females show positive association; $t=4.42, p=0.003$ and $t=3.66$, $p=0.001$ ), for $\mathrm{P}$ in the posterior cingulate gyrus (females show a negative association; $t=4.72, p=0.006)$, the medial superior frontal gyrus (females show a negative association; $t=4.62$, $p=0.006$ ), and the middle cingulate gyrus (females show a negative association; $t=4.56, p=0.001$ ). See Figure 2 .

\section{Within-Sex Correlation Between Temperaments and Voxel-Wise GMv}

To investigate the correlation between temperaments and voxelwise GMv for males and females separately, we performed

\footnotetext{
${ }^{4}$ http://www.alivelearn.net/xjview

${ }^{5}$ http://www.mccauslandcenter.sc.edu/mricro/mricron
} 


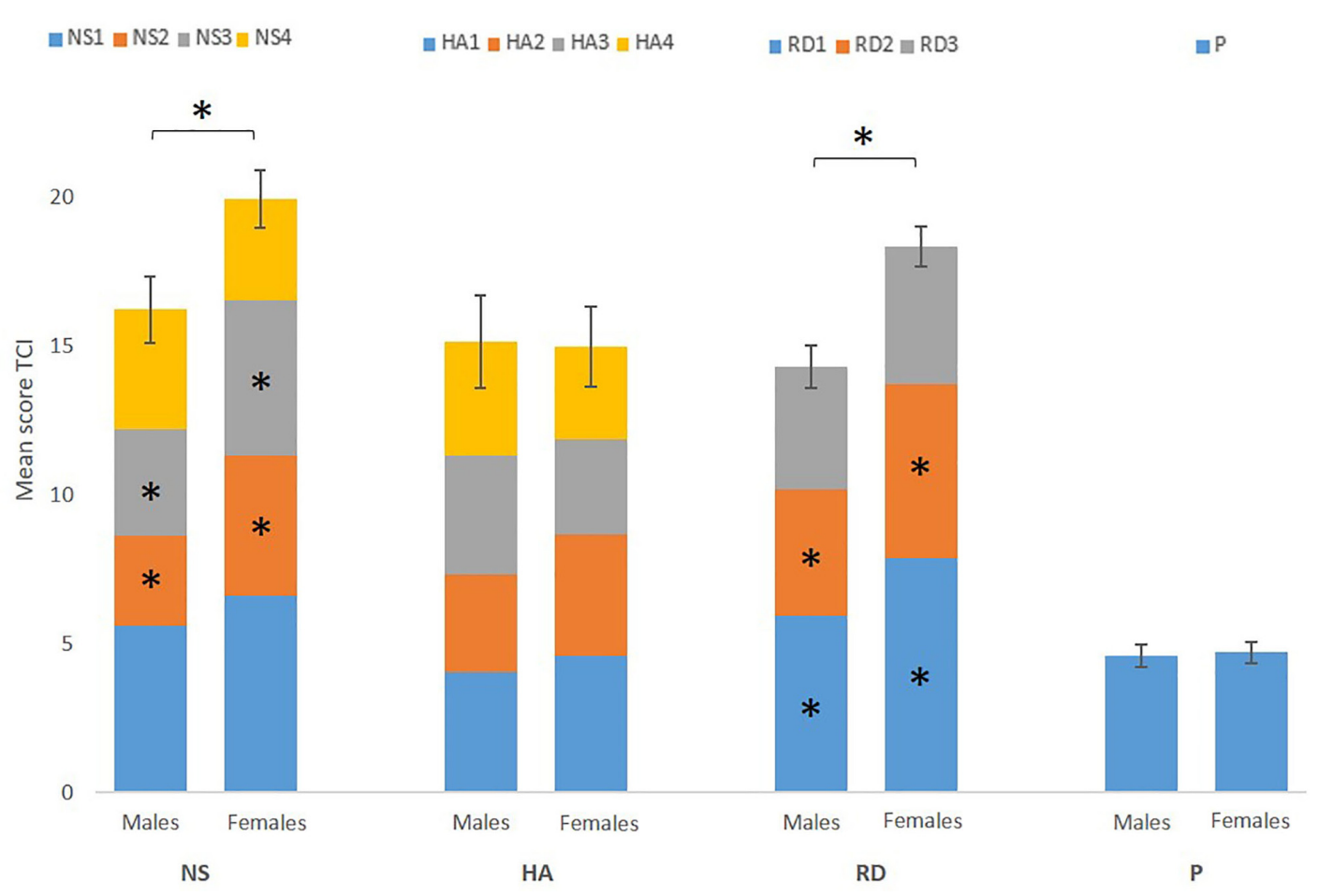

FIGURE 1 | Mean score of the four temperament scores [novelty seeking (NS), harm avoidance (HA), reward dependence (RD), and persistence (P)] and each subscale (NS1, NS2, NS3, NS4, HA1, HA2, HA3, HA4, RD1, RD2, RD3, and P) for males and females separately. Error bars represent standard error of the total score (NS, HA, RD, and P). The results show a significant higher score on NS and RD for females, driven by NS2, NS3, RD1, and RD2. *Marks significance at $p<0.05$.

TABLE 1 | Sex-temperament interaction effects in voxel-wise GMv.

\begin{tabular}{|c|c|c|c|c|c|c|c|c|c|}
\hline & \multirow[t]{2}{*}{ Area } & \multirow[t]{2}{*}{$P$} & \multirow[t]{2}{*}{$R / L$} & \multirow[t]{2}{*}{ \#Voxels } & \multirow[t]{2}{*}{$Z$-value } & \multirow[t]{2}{*}{$t$-value } & \multicolumn{3}{|c|}{ Coordinates } \\
\hline & & & & & & & $\mathbf{x}$ & $\mathbf{y}$ & $\mathbf{z}$ \\
\hline NS & Superior temporal gyrus & 0.001 & $\mathrm{R}$ & 12 & 4.51 & 4.96 & 56 & -44 & 18 \\
\hline $\mathrm{HA}$ & Middle temporal gyrus & 0.003 & $L$ & 10 & 4.56 & 5.04 & -50 & -74 & 23 \\
\hline \multirow[t]{2}{*}{$\mathrm{RD}$} & Insula & 0.003 & $\mathrm{R}$ & 11 & 4.42 & 4.09 & 35 & -8 & 9 \\
\hline & Insula & 0.001 & $\mathrm{R}$ & 15 & 3.66 & 3.45 & 35 & 17 & -14 \\
\hline \multirow[t]{3}{*}{$P$} & Posterior cingulate gyrus & 0.006 & $\mathrm{R}$ & 12 & 4.72 & 5.25 & 5 & -36 & 29 \\
\hline & Medial superior frontal gyrus & 0.006 & $\mathrm{~L}$ & 12 & 4.62 & 5.11 & 11 & 44 & 48 \\
\hline & Middle cingulate gyrus & 0.001 & $\mathrm{~L}$ & 18 & 4.56 & 5.03 & 0 & -32 & 36 \\
\hline
\end{tabular}

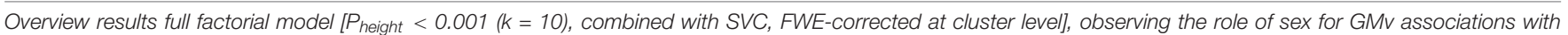
temperament traits of the TCl; novelty seeking (NS), harm avoidance (HA), reward dependence (RD), and persistence (P). Coordinates refer to MNI-space.

two separate multiple regression analyses on the smoothed GM-images. The results are shown in Table 2. To investigate any overlap between the sex-selective results, we inclusively masked the results of the regression analysis of both groups. No overlap was observed.

\section{DISCUSSION}

In the current study, we investigated sex differences in temperament-brain associations, as well as shared temperamentbrain associations between the sexes.

\section{Sex Differences in Temperament Traits}

We observed significant sex differences in NS and RD between males and females. For both temperaments, females had a significantly higher score than males.

Extraversion, is a trait of the Big Five that is known to be linked with NS (Gocłowska et al., 2018). A study by Weisberg et al. (2011) observed significantly higher overall extraversion score in females. Furthermore, individuals scoring high with respect to NS tend to be enthusiastic, impulsive, and NS is known to be linked to the neurotransmitter dopamine (Cloninger, 1987; Larsen and Buss, 2010). Previous research has shown that females score higher in enthusiasm 


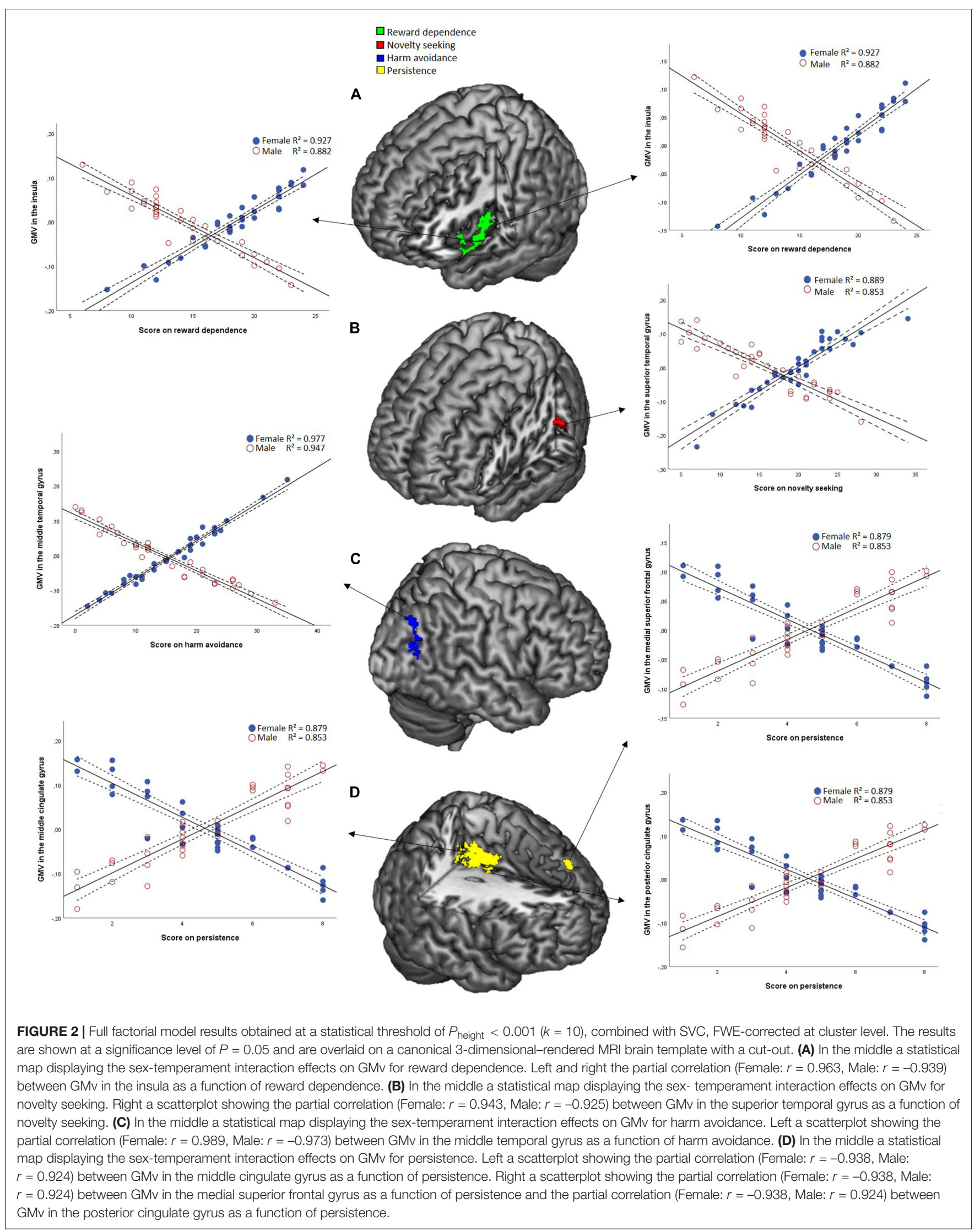


TABLE 2 | Within-sex correlation between temperaments and voxel-wise GMv.

\begin{tabular}{|c|c|c|c|c|c|c|c|c|c|c|}
\hline & \multirow[t]{2}{*}{ Area } & \multirow{2}{*}{$\begin{array}{c}r \\
+/-\end{array}$} & \multirow[t]{2}{*}{$P$} & \multirow[t]{2}{*}{$R / L$} & \multirow[t]{2}{*}{ \#Voxels } & \multirow[t]{2}{*}{$Z$-value } & \multirow[t]{2}{*}{$t$-value } & \multicolumn{3}{|c|}{ Coordinates } \\
\hline & & & & & & & & $\mathbf{x}$ & $\mathbf{y}$ & $\mathbf{z}$ \\
\hline \multirow[t]{5}{*}{ NS Female } & Caudate nucleus & + & $<0.001$ & $\mathrm{R}$ & 22 & 4.24 & 5.16 & 3 & 9 & -6 \\
\hline & Thalamus & + & 0.002 & $L$ & 10 & 3.68 & 4.27 & -45 & -62 & 50 \\
\hline & Angular gyrus & + & 0.001 & $L$ & 13 & 3.55 & 4.07 & -18 & -26 & 11 \\
\hline & Anterior cingulate sulcus & - & $<0.001$ & $\mathrm{~L}$ & 20 & 3.60 & 4.14 & 0 & 32 & 11 \\
\hline & Mid frontal gyrus & - & $<0.001$ & $L$ & 30 & 4.32 & 5.29 & -35 & 2 & 62 \\
\hline \multirow[t]{2}{*}{ NS Male } & Rolandic operculum & + & $<0.001$ & $\mathrm{R}$ & 69 & 4.28 & 5.13 & 53 & 5 & 2 \\
\hline & Precentral gyrus & + & $<0.001$ & $\mathrm{R}$ & 21 & 4.22 & 5.22 & 30 & -9 & 56 \\
\hline \multirow[t]{7}{*}{ HA Female } & Inferior frontal gyrus & + & 0.002 & $L$ & 14 & 4.20 & 5.09 & -18 & 32 & -24 \\
\hline & Fusiform gyrus & + & 0.001 & $L$ & 16 & 4.31 & 5.28 & -44 & -65 & -18 \\
\hline & Superior temporal sulcus & + & 0.001 & $\mathrm{R}$ & 15 & 3.91 & 4.62 & 60 & 2 & -5 \\
\hline & Superior temporal sulcus & + & 0.002 & $L$ & 13 & 4.39 & 5.40 & -65 & -39 & 11 \\
\hline & Mid temporal sulcus & + & 0.004 & $L$ & 11 & 4.01 & 4.78 & -59 & -51 & 14 \\
\hline & Supramarginal gyrus & + & $<0.001$ & $\mathrm{R}$ & 19 & 4.04 & 4.82 & 63 & -24 & 33 \\
\hline & Cerebellum (L9) & - & $<0.001$ & $\mathrm{R}$ & 15 & 3.80 & 4.45 & 8 & -57 & -59 \\
\hline \multirow[t]{2}{*}{ HA Male } & Rolandic operculum & + & $<0.001$ & $\mathrm{R}$ & 25 & 4.14 & 4.99 & 53 & 5 & 2 \\
\hline & Mid cingulate sulcus & + & $<0.001$ & $\mathrm{R}$ & 17 & 3.51 & 4.02 & 5 & 5 & 30 \\
\hline \multirow[t]{8}{*}{ RD Female } & Cerebellum Crus1 & + & $<0.001$ & $L$ & 30 & 3.87 & 4.56 & -33 & -78 & -29 \\
\hline & Cerebellum Crus1 & + & 0.001 & $L$ & 15 & 3.78 & 4.42 & -24 & -84 & -27 \\
\hline & Cerebellum Crus1 & + & 0.003 & $L$ & 12 & 3.54 & 4.06 & -21 & -80 & -27 \\
\hline & Frontal inferior orbital gyrus & + & 0.001 & $L$ & 17 & 3.96 & 4.65 & -24 & 24 & -20 \\
\hline & Insula & + & 0.004 & $\mathrm{R}$ & 11 & 4.15 & 5.01 & 35 & -9 & 11 \\
\hline & Fusiform gyrus & - & 0.001 & $L$ & 15 & 3.49 & 3.99 & -39 & -60 & -21 \\
\hline & Insula & - & 0.002 & $L$ & 11 & 3.58 & 4.11 & -38 & -11 & -6 \\
\hline & Insula & - & 0.002 & $L$ & 11 & 3.78 & 4.41 & -36 & -30 & 21 \\
\hline RD Male & - & & & & & & & & & \\
\hline \multirow[t]{7}{*}{ P Female } & Cerebellum (L7b) & - & $<0.001$ & $L$ & 161 & 4.77 & 6.12 & -44 & -53 & -53 \\
\hline & Parahippocampal gyrus & - & $<0.001$ & $L$ & 35 & 4.28 & 5.22 & -18 & -26 & -20 \\
\hline & Inferior frontal gyrus & - & $<0.001$ & $\mathrm{R}$ & 53 & 5.04 & 6.66 & 21 & 12 & -18 \\
\hline & Thalamus & - & $<0.001$ & $\mathrm{R}$ & 33 & 4.19 & 5.07 & 11 & -30 & 9 \\
\hline & Posterior cingulate gyrus & - & $<0.001$ & $\mathrm{R}$ & 56 & 4.46 & 5.54 & 5 & -51 & 11 \\
\hline & Parahippocampal gyrus & - & 0.001 & $\mathrm{R}$ & 17 & 4.45 & 5.53 & 17 & -41 & -9 \\
\hline & Inferior Frontal gyrus & - & $<0.001$ & $L$ & 24 & 4.13 & 4.98 & -29 & 38 & -14 \\
\hline
\end{tabular}

P Male

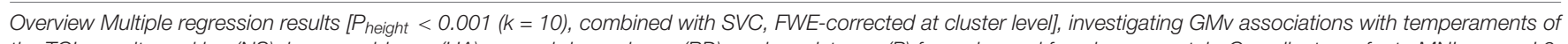

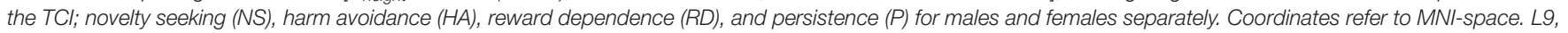
lobe 9; $L 7 b$, lobe $7 b$.

(Costa et al., 2001; Weisberg et al., 2011) and that estradiol, the female sex hormone, modulates mesolimbic dopamine systems and so affects motivated behaviors (Yoest et al., 2014). On the other hand, no associations between the total scores of NS and total testosterone, the male sex hormone, have been found (Tsuchimine et al., 2015). Previous studies have looked at sex differences in a previous version of the TCI, the Tridimensional Personality Questionnaire (TPQ) (Cloninger et al., 1991). However, these studies found conflicting findings on NS score and impulsivity when looking at sex differences (Reynolds et al., 2006; Mitchell and Potenza, 2015). A possible explanation for these conflicting findings on impulsivity and NS, may be that females show fluctuating levels of impulsivity due to the menstrual cycle and changing estrogen levels (Weafer and de Wit, 2014). Furthermore, when looking at the different subscales of NS, we found that the impulsiveness (NS2) $(p=0.029)$ and the extravagance (NS3) $(p=0.005)$ dimensions of NS specifically drive the significant sex differences in NS. A study using the TPQ (Cloninger, 1987) also found a significantly higher score for females on NS3 (Zohar et al., 2001). They also found a positive correlation between NS3 and RD, the second temperament where we found a significant higher score for females than for males.

Reward dependence has been shown to be linked with norepinephrine, previous research has already shown that through the central nervous system estrogen can modulate noradrenergic neurotransmission (Vega-Rivera et al., 2013). Furthermore, our findings on $\mathrm{RD}$ are in line with previous research (Cloninger et al., 1991; Zohar et al., 2001) and showed that for $\mathrm{RD}$ the significant sex difference was 
driven by sentimentality (RD1) $(p<0.001)$ and attachment (RD2) $(p=0.010)$. RD is often described as inter-personal sensitivity and sociability. Generally, females focus more on interpersonal relationships, score higher on attachment, warmth and empathy (Zohar et al., 2001; Weisberg et al., 2011; Weafer and de Wit, 2014) and are more concerned with the opinion of others in social tasks than males (Cloninger et al., 1991; Vega-Rivera et al., 2013). Males tend to focus more on individuality and achievement (Sato and McCann, 1998). These results provide support for the higher score for females on RD.

In summary, we found significantly higher scores for females on NS and RD. These results indicate that mainly for the temperaments linked to sociability and attachment, we find a significant higher score for females.

\section{Interaction Between Sex and Temperaments on Voxel-Wise GMv}

We found opposite associations in both groups between GMv in the superior temporal gyrus and NS, with a positive association for females. Previous research found a significant positive correlation between NS and glucose metabolism in the superior temporal gyrus (Hakamata et al., 2006), which is a region that is linked with impulsivity and social cognition (Horn et al., 2003; Grecucci et al., 2013). Furthermore, a previous study showed that females mainly have more GM percentage in the superior temporal gyrus than males (Schlaepfer, 1995). A study looking at the difference between pre- and post-menopausal females, showed a decrease in GMv in postmenopausal females. The GMv was also found to be positively correlated to estradiol, the major female sex hormone (Kim et al., 2018). In contrast with our hypothesis we did not find any results for NS that were comparable to the study of Nostro et al. (2016). A possible explanation for this discrepancy may relate to the methods (Multiple regression analysis vs. full factorial model analysis). Alternatively, the similarity between NS and extraversion may be limited.

Secondly, we observed inverse associations in both groups between GMv in the insula and RD, with a positive association in females. $\mathrm{RD}$ is associated with responsiveness to signals of reward, the insula is known to play a part in the additional reward-sensitive brain areas (O'Doherty et al., 2002; Kirsch et al., 2003). Research shows that females show more GMv in the right insula (Ruigrok et al., 2014) and estrogen is found to excite neurons in the insula (Saleh et al., 2004). The insula is part of the limbic system. Previous research has shown that females have a larger limbic volume (Goldstein et al., 2001; Zaidi, 2010). It has been proposed that due to a larger limbic brain females are better in touch with their emotions and can better connect to others (Zaidi, 2010).

Thirdly, we found opposite associations between HA and GMv in the middle temporal gyrus. There are contradictory findings about the link between HA and the middle temporal gyrus (Hakamata et al., 2006; Iidaka et al., 2006). As previous studies have shown both positive (Iidaka et al., 2006) and negative correlations (Hakamata et al., 2006) between HA and the middle temporal gyrus. The middle temporal gyri is also known to be linked to social cognition (Grecucci et al., 2013). Furthermore, we did not find a significant sex difference in HA score.

$\mathrm{P}$ is the only temperament with opposite associations for which we found a positive association for males. We did not find a sex difference for score in P. P indicates motivation without direct external reward (Cloninger et al., 1993). The medial superior frontal gyrus and the cingulate gyri are areas known to be involved in cognitive control and motivation (Heilbronner et al., 2011; Bahlmann et al., 2015).

In summary, the results show that GMv of the superior temporal gyrus, middle temporal gyrus, and insula show a positive association between temperaments and regional GMv in females and a negative association in males, while a positive association in males and a negative association in females was observed between $\mathrm{P}$ and regional GMv in the posterior cingulate gyrus, medial superior frontal gyrus, and middle cingulate gyrus.

\section{Within-Sex Correlation Between Temperaments and Voxel-Wise GMv}

We found non-overlapping sex-specific topographic patterns in temperament-brain associations. These results suggests that the structural neurobiology underlying personality is to a high degree sex-specific and our results are in line with the study of Nostro et al. (2016), who only found sex-specific associations. Our results support their hypothesis that brain structure-personality associations are highly dependent on sex and this may be attributable to hormonal interplays.

\section{Clinical Implications}

Different temperaments from the TCI have been linked to several neuropsychiatric disorders; NS is known to be correlated with drug addiction (Bardo et al., 1996; Lin et al., 2015; Vanhille et al., 2015), tobacco abuse (Palmer et al., 2013), and depression (Duclot and Kabbaj, 2013). NS and HA are both linked to pathological gambling (Kim and Grant, 2001; Nordin and Nylander, 2007) and alcohol abuse (Palmer et al., 2013; Wennberg et al., 2014). However, the risk for these neuropsychiatric symptoms differs between males and females. For example, studies show that males have a higher risk for developing an alcohol or gambling addiction (Engwall et al., 2004; Nolen-Hoeksema and Hilt, 2006). Our results support the importance of sex in the neurobiology of these disorders.

\section{Limitations}

A limitation of the current study is the small sample size. However, the different subgroups that constituted the sample presumably increased the variability of the dataset and benefited the statistical power. Furthermore, we used a statistical threshold $\left[P_{\text {height }}<0.001(k=10)\right.$ in combination with $P_{\text {height }}<0.05$ FWEcorrected following Small Volume Correction]. However, as the current study is an exploratory study, further studies are needed with larger sample sizes. It is important to keep in mind that females generally have smaller brains than males (Ruigrok et al., 2014) and this can effect volume of specific brain regions (Pintzka et al., 2015). To control for this, TIV was entered as variable of no 
interest in all analyses. Furthermore, as the study is correlational in nature, any causal interpretations are unjustified.

\section{CONCLUSION}

The present study documents opposing associations in males and females between temperament brain associations. The results reveal that sex-specific associations outweigh sex-general associations in the neurobiology of personality.

\section{ETHICS STATEMENT}

This study was approved by the Ethical Committee of University Hospitals Leuven. All subjects gave written

\section{REFERENCES}

Afifi, M. (2007). Gender differences in mental health. Singapore Med. J. 48, 385-391.

Bahlmann, J., Aarts, E., and D'Esposito, M. (2015). Influence of motivation on control hierarchy in the human frontal cortex. J. Neurosci. 35, 3207-3217. doi: 10.1523/JNEUROSCI.2389-14.2015

Bardo, M. T., Donohew, R. L., and Harrington, N. G. (1996). Psychobiology of novelty seeking and drug seeking behavior. Behav. Brain Res. 77, 23-43. doi: 10.1016/0166-4328(95)00203-0

Chapman, B. P., Duberstein, P. R., Sörensen, S., and Lyness, J. M. (2007). Gender differences in five factor model personality traits in an elderly cohort. Pers. Individ. Differ. 43, 1594-1603. doi: 10.1016/j.paid.2007.04.028

Cloninger, C. R. (1986). A unified biosocial theory of personality and its role in the development of anxiety states. Psychiatr. Dev. 4, 167-226.

Cloninger, C. R. (1987). A systematic method for clinical description and classification of personality variants. A proposal. Arch. Gen. Psychiatry 44, 573-588.

Cloninger, C. R., Przybeck, T. R., and Svrakic, D. M. (1991). The tridimensional personality questionnaire: U.S. normative data. Psychol. Rep. 69(3 Pt 1), 1047-1057. doi: 10.2466/pr0.1991.69.3.1047

Cloninger, C. R., Svrakic, D. M., and Przybeck, T. R. (1993). A psychobiological model of temperament and character. Arch. Gen. Psychiatry 50, 975-990.

Comings, D. E., Gade-Andavolu, R., Gonzalez, N., Wu, S., Muhleman, D., Blake, H., et al. (2000). A multivariate analysis of 59 candidate genes in personality traits: the temperament and character inventory. Clin. Genet. 58, 375-385. doi: 10.1034/j.1399-0004.2000.580508.x

Costa, P. T., and McCrae, R. (1992a). Normal personality assessment in clinical practice: the NEO personality inventory. Psychol. Assess. 4, 5-13. doi: 10.1037/ /1040-3590.4.1.5

Costa, P. T., and McCrae, R. (1992b). Professional Manual: Revised NEO Personality Inventory (NEO-PI-R) and NEO Five-Factor Inventory (NEO-FFI). Odessa, FL: Psychological Assessment Resources.

Costa, P. T., Terracciano, A., and McCrae, R. R. (2001). Gender differences in personality traits across cultures: robust and surprising findings. J. Pers. Soc. Psychol. 81, 322-331. doi: 10.1037/0022-3514.81.2.322

Duclot, F., and Kabbaj, M. (2013). Individual differences in novelty seeking predict subsequent vulnerability to social defeat through a differential epigenetic regulation of brain-derived neurotrophic factor expression. J. Neurosci. 33, 11048-11060. doi: 10.1523/JNEUROSCI.0199-13.2013

Engwall, D., Hunter, R., and Steinberg, M. (2004). Gambling and other risk behaviors on university campuses. J. Am. Coll. Health 52, 245-256. doi: 10.3200/ JACH.52.6.245-256

Gardini, S., Cloninger, C. R., and Venneri, A. (2009). Individual differences in personality traits reflect structural variance in specific brain regions. Brain Res. Bull. 79, 265-270. doi: 10.1016/j.brainresbull.2009.03.005 informed consent in accordance with the Declaration of Helsinki.

\section{AUTHOR CONTRIBUTIONS}

DS and JVdS contributed conception and design of the study and organized the database. DS performed the statistical analysis and wrote the first draft of the manuscript. Y-AH analyzed the data. All authors contributed to manuscript revision, read, and approved the submitted version.

\section{FUNDING}

JVdS was supported by a KU Leuven Starting Grant.

Gaser, C., and Kurth, F. (2017). Manual Computational Anatomy Toolbox-CAT12. Jena: University of Jena.

Gocłowska, M. A., Ritter, S. M., Elliot, A. J., and Baas, M. (2018). Novelty seeking is linked to openness and extraversion, and can lead to greater creative performance. J. Pers. 87, 252-266. doi: 10.1111/jopy.12387

Goldstein, J. M., Seidman, L. J., Horton, N. J., Makris, N., Kennedy, D. N., Caviness, V. S., et al. (2001). Normal sexual dimorphism of the adult human brain assessed by in vivo magnetic resonance imaging. Cereb. Cortex 11, 490-497. doi: 10.1093/cercor/11.6.490

Grecucci, A., Giorgetta, C., Bonini, N., and Sanfey, A. G. (2013). Reappraising social emotions: the role of inferior frontal gyrus, temporo-parietal junction and insula in interpersonal emotion regulation. Front. Hum. Neurosci. 7:523. doi: 10.3389/fnhum.2013.00523

Gual, P., Pérez-Gaspar, M., Martínez-González, M. A., Lahortiga, F., de IralaEstévez, J., and Cervera-Enguix, S. (2002). Self-esteem, personality, and eating disorders: baseline assessment of a prospective population-based cohort. Int. J. Eat. Disord. 31, 261-273. doi: 10.1002/eat.10040

Hakamata, Y., Iwase, M., Iwata, H., Kobayashi, T., Tamaki, T., Nishio, M., et al. (2006). Regional brain cerebral glucose metabolism and temperament: a positron emission tomography study. Neurosci. Lett. 396, 33-37. doi: 10.1016/j. neulet.2005.11.017

Heath, A. C., Cloninger, C. R., and Martin, N. G. (1994). Testing a model for the genetic structure of personality: a comparison of the personality systems of Cloninger and Eysenck. J. Pers. Soc. Psychol. 66, 762-775. doi: 10.1037//00223514.66.4.762

Heilbronner, S. R., Hayden, B. Y., and Platt, M. L. (2011). Decision salience signals in posterior cingulate cortex. Front. Neurosci. 5:55. doi: 10.3389/fnins.2011. 00055

Horn, N. R., Dolan, M., Elliott, R., Deakin, J. F. W., and Woodruff, P. W. R. (2003). Response inhibition and impulsivity: an fMRI study. Neuropsychologia 41, 1959-1966. doi: 10.1016/S0028-3932(03)00077-0

Iidaka, T., Matsumoto, A., Ozaki, N., Suzuki, T., Iwata, N., Yamamoto, Y., et al. (2006). Volume of left amygdala subregion predicted temperamental trait of harm avoidance in female young subjects. A voxel-based morphometry study. Brain Res. 1125, 85-93. doi: 10.1016/j.brainres.2006. 09.015

Kim, G.-W., Park, K., and Jeong, G.-W. (2018). Effects of sex hormones and age on brain volume in post-menopausal women. J. Sex. Med. 15, 662-670. doi: 10.1016/j.jsxm.2018.03.006

Kim, S. W., and Grant, J. E. (2001). Personality dimensions in pathological gambling disorder and obsessive-compulsive disorder. Psychiatry Res. 104, 205-212. doi: 10.1016/s0165-1781(01)00327-4

Kirsch, P., Schienle, A., Stark, R., Sammer, G., Blecker, C., Walter, B., et al. (2003). Anticipation of reward in a nonaversive differential conditioning paradigm and the brain reward system. Neuroimage 20, 1086-1095. doi: 10.1016/S10538119(03)00381-1 
Kret, M. E., and De Gelder, B. (2012). A review on sex differences in processing emotional signals. Neuropsychologia 50, 1211-1221. doi: 10.1016/j. neuropsychologia.2011.12.022

Krug, I., Pinheiro, A. P., Bulik, C., Jiménez-Murcia, S., Granero, R., Penelo, E., et al. (2009). Lifetime substance abuse, family history of alcohol abuse/dependence and novelty seeking in eating disorders: comparison study of eating disorder subgroups. Psychiatry Clin. Neurosci. 63, 82-87. doi: 10.1111/j.1440-1819.2008. 01908.x

Laricchiuta, D., Petrosini, L., Piras, F., Macci, E., Cutuli, D., Chiapponi, C., et al. (2014). Linking novelty seeking and harm avoidance personality traits to cerebellar volumes: personality traits and cerebellar volumes. Hum. Brain Mapp. 35, 285-296. doi: 10.1002/hbm.22174

Larsen, R. J., and Buss, D. M. (2010). Personality Psychology: Domains of Knowledge About Human Nature, 4th Edn. New York, NY: McGraw-Hill Companies.

Lin, S.-H., Lee, L.-T., Tsai, H. C., Chen, K. C., Chen, W. T., Lee, I. H., et al. (2015). Association between blood level of plasma oxytocin and novelty seeking among methadone-maintained heroin users. Neuropsychobiology 71, 65-69. doi: $10.1159 / 000371637$

Mitchell, M. R., and Potenza, M. N. (2015). Importance of sex differences in impulse control and addictions. Front. Psychiatry 6:24. doi: 10.3389/fpsyt.2015. 00024

Nolen-Hoeksema, S., and Hilt, L. (2006). Possible contributors to the gender differences in alcohol use and problems. J. Gen. Psychol. 133, 357-374. doi: 10.3200/GENP.133.4.357-374

Nordin, C., and Nylander, P.-O. (2007). Temperament and character in pathological gambling. J. Gambl. Stud. 23, 113-120. doi: 10.1007/s10899-0069049-x

Nostro, A. D., Müller, V. I., Reid, A. T., and Eickhoff, S. B. (2016). Correlations between personality and brain structure: a crucial role of gender. Cereb. Cortex 27, 3698-3712. doi: 10.1093/cercor/bhw191

O’Doherty, J. P., Deichmann, R., Critchley, H. D., and Dolan, R. J. (2002). Neural responses during anticipation of a primary taste reward. Neuron 33, 815-826. doi: 10.1016/s0896-6273(02)00603-7

Palmer, R. H. C., Knopik, V. S., Rhee, S. H., Hopfer, C. J., Corley, R. C., Young, S. E., et al. (2013). Prospective effects of adolescent indicators of behavioral disinhibition on DSM-IV alcohol, tobacco, and illicit drug dependence in young adulthood. Addict. Behav. 38, 2415-2421. doi: 10.1016/j.addbeh.2013.03.021

Picerni, E., Petrosini, L., Piras, F., Laricchiuta, D., Cutuli, D., Chiapponi, C., et al. (2013). New evidence for the cerebellar involvement in personality traits. Front. Behav. Neurosci. 7:133. doi: 10.3389/fnbeh.2013.00133

Pintzka, C. W. S., Hansen, T. I., Evensmoen, H. R., and Håberg, A. K. (2015). Marked effects of intracranial volume correction methods on sex differences in neuroanatomical structures: a HUNT MRI study. Front. Neurosci. 9:238. doi: 10.3389/fnins.2015.00238

Raine, A., Yang, Y., Narr, K. L., and Toga, A. W. (2011). Sex differences in orbitofrontal gray as a partial explanation for sex differences in antisocial personality. Mol. Psychiatry 16, 227-236. doi: 10.1038/mp.2009.136

Reynolds, B., Ortengren, A., Richards, J. B., and de Wit, H. (2006). Dimensions of impulsive behavior: personality and behavioral measures. Pers. Individ. Differ. 40, 305-315. doi: 10.1016/j.paid.2005.03.024

Ruigrok, A. N. V., Salimi-Khorshidi, G., Lai, M.-C., Baron-Cohen, S., Lombardo, M. V., Tait, R. J., et al. (2014). A meta-analysis of sex differences in human brain structure. Neurosci. Biobehav. Rev. 39, 34-50. doi: 10.1016/j.neubiorev.2013. 12.004

Saleh, T. M., Connell, B. J., Legge, C., and Cribb, A. E. (2004). Estrogen attenuates neuronal excitability in the insular cortex following middle cerebral artery occlusion. Brain Res. 1018, 119-129. doi: 10.1016/j.brainres.2004.05.074
Sato, T., and McCann, D. (1998). Individual differences in relatedness and individuality: an exploration of two constructs. Pers. Individ. Differ. 24, 847-859. doi: 10.1016/S0191-8869(98)00020-8

Schlaepfer, T. (1995). Structural differences in the cerebral cortex of healthy female and male subjects: a magnetic resonance imaging study. Psychiatry Res. Neuroimaging 61, 129-135. doi: 10.1016/0925-4927(95) 02634-A

Stallings, M. C., Hewitt, J. K., Cloninger, C. R., Heath, A. C., and Eaves, L. J. (1996). Genetic and environmental structure of the tridimensional personality questionnaire: three or four primary temperament dimensions. J. Pers. Soc. Psychol. 70, 127-140. doi: 10.1037//0022-3514.70.1.127

Stam, D., Huang, Y.-A., and Van den Stock, J. (2018). Gray matter volume of a region in the thalamic pulvinar is specifically associated with novelty seeking. Front. Psychol. 9:203. doi: 10.3389/fpsyg.2018.00203

Tsuchimine, S., Kaneda, A., Nakamura, K., and Yasui-Furukori, N. (2015). The relationships between androgens and novelty seeking in healthy Japanese men. Psychiatry Res. 225, 175-178. doi: 10.1016/j.psychres.2014. 11.013

Vanhille, N., Belin-Rauscent, A., Mar, A. C., Ducret, E., and Belin, D. (2015). High locomotor reactivity to novelty is associated with an increased propensity to choose saccharin over cocaine: new insights into the vulnerability to addiction. Neuropsychopharmacology 40, 577-589. doi: 10.1038/npp.2014.204

Vega-Rivera, N. M., López-Rubalcava, C., Paez-Martínez, N., Castro, M., and Estrada-Camarena, E. M. (2013). Interacción estrógenos-noradrenalina en la depresión. Salud Mental 36, 331-336. doi: 10.17711/SM.0185-3325.2013.040

Weafer, J., and de Wit, H. (2014). Sex differences in impulsive action and impulsive choice. Addict. Behav. 39, 1573-1579. doi: 10.1016/j.addbeh.2013.10.033

Weisberg, Y. J., DeYoung, C. G., and Hirsh, J. B. (2011). Gender differences in personality across the ten aspects of the big five. Front. Psychol. 2:178. doi: $10.3389 /$ fpsyg. 2011.00178

Wennberg, P., Berglund, K., Berggren, U., Balldin, J., and Fahlke, C. (2014). The cloninger Type I/Type II typology: configurations and personality profiles in socially stable alcohol dependent patients. Adv. Psychiatry 3, 1-5. doi: 10.1155/ 2014/346157

Yang, W., Cun, L., Du, X., Yang, J., Wang, Y., Wei, D., et al. (2015). Gender differences in brain structure and resting-state functional connectivity related to narcissistic personality. Sci. Rep. 5:10924. doi: 10.1038/srep10924

Yoest, K. E., Cummings, J. A., and Becker, J. B. (2014). Estradiol, dopamine and motivation. Cent. Nerv. Syst. Agents Med. Chem. 14, 83-89. doi: 10.2174/ 1871524914666141226103135

Zaidi, Z. F. (2010). Gender differences in human brain: a review. Open Anat. J. 2, 37-55. doi: 10.2174/1877609401002010037

Zohar, A., Lev-Ari, L., Benjamin, J., Ebstein, R., Lichtenberg, P., and Osher, Y. (2001). The psychometric properties of the Hebrew version of Cloninger's tridimensional personality questionnaire. Pers. Individ. Differ. 30, 1297-1309. doi: 10.1016/S0191-8869(00)00112-4

Conflict of Interest Statement: The authors declare that the research was conducted in the absence of any commercial or financial relationships that could be construed as a potential conflict of interest.

Copyright (c) 2019 Stam, Huang and Van den Stock. This is an open-access article distributed under the terms of the Creative Commons Attribution License (CC BY). The use, distribution or reproduction in other forums is permitted, provided the original author(s) and the copyright owner(s) are credited and that the original publication in this journal is cited, in accordance with accepted academic practice. No use, distribution or reproduction is permitted which does not comply with these terms. 